\title{
Uso de un diseño de mezclas para el desarrollo de hamburguesas de carne de bovino, con un perfil nutricional mejorado con harina de linaza, pulpa de mango y ciruela deshidratada
}

\author{
Usage of a mixture design for the development of beef patties, with an improved nutritional profile \\ with flaxseed flour, mango pulp, and dried plum
}

\begin{abstract}
Carlos Montalvo-Navarro', Germán Cumplido-Barbeitia', Humberto González-Ríos', Luz del Carmen MontoyaBallesteros ${ }^{2}$, Anna Judith Pérez-Báez ${ }^{1}$, Libertad Zamorano-García ${ }^{1}$, Martín Valenzuela-Melendres ${ }^{1 *}$

Coordinación de Tecnología de Alimentos de Origen Animal, Centro de Investigación en Alimentación y Desarrollo. Carretera Gustavo Enrique Astiazarán Rosas, No. 46, Col. La Victoria, CP 83304, Hermosillo, Sonora, México.

2 Coordinación de Tecnología de Alimentos de Origen Vegetal, Centro de Investigación en Alimentación y Desarrollo. Carretera Gustavo Enrique Astiazarán Rosas, No. 46, Col. La Victoria, CP 83304, Hermosillo, Sonora, México.
\end{abstract}

\section{RESUMEN}

Se utilizó la metodología de superficie de respuesta para estudiar los efectos de harina de linaza $(\mathrm{HL})$, pulpa de mango (PM) y ciruela deshidratada (CD) sobre la calidad fisicoquímica y sensorial de hamburguesas de carne de bovino. Además, se optimizó su incorporación en la formulación cárnica para incrementar el contenido de ácidos grasos poliinsaturados, sin demérito en su calidad, usando para ello un diseño de mezclas. De acuerdo a los modelos de regresión obtenidos, la adición de $\mathrm{HL}$ y PM aumentó el color instrumental $(p<0.05)$, mientras que la CD lo disminuyó. La acidez aumentó por la adición de PM o CD, y el rendimiento aumentó por la adición de HL y CD. La incorporación de PM o CD incrementó las propiedades sensoriales y los valores de textura, mientras que la $\mathrm{HL}$ tuvo un efecto contrario. La fórmula óptima incluyó $6 \% \mathrm{HL}, 10.5 \%$ PM y $3.5 \%$ CD, y tuvo mayor contenido de ácidos grasos polinsaturados, principalmente omega-3. Las hamburguesas de carne de bovino adicionadas con $\mathrm{HL}, \mathrm{PM}$ y CD son una alternativa de alimentación más saludable para la población. Son necesarios estudios posteriores que demuestren el impacto de su consumo en la salud de las personas.

Palabras clave: Hamburguesa de carne de bovino, productos cárnicos funcionales, desarrollo de nuevos productos

\section{ABSTRACT}

The response surface methodology was used to study the effects of flaxseed flour (FS), mango pulp (MP), and dried prune (DP), on the physicochemical and sensory properties of beef patties. In addition, its incorporation into the meat formulation was optimized to increase the content of polyunsaturated fatty acids without demerit in their quality properties, by using a mixture design. According to the obtained models, FS and MP addition increased the instrumental color values $(p<0.05)$, while DP decreased them. The $\mathrm{pH}$ values decreased by the addition of MP or DP, and cooking yield increased by the addition of FS and DP. The incorporation of MP or DP increased sensory properties and

*Autor para correspondencia: Martín Valenzuela Melendres

Correo electrónico: martin@ciad.mx

Recibido: 5 de julio de 2021

Aceptado: 1 de diciembre de 2021 texture values, while FS had a contrary effect. The optimized formulation included $6 \% \mathrm{FS}, 10.5 \% \mathrm{MP}$ and $3.5 \% \mathrm{DP}$, which resulted in a higher amount of polyunsaturated fatty acids, mainly omega-3. Beef patties formulated with FS, MP, and DP could be a healthier food alternative for the population. Further studies are necessary to demonstrate the impact of its consumption on the health of consumers.

Keywords: beef patties, functional meat products, new products development

\section{INTRODUCCIÓN}

La producción de carne tiene un peso importante en la economía de México, ya que en 2017 esta actividad se posicionó en el primer lugar dentro del valor de la producción agropecuaria. Los mexicanos consumen en promedio $69 \mathrm{~kg}$ de carne, destacando el pollo, cerdo y res (Comecarne, 2021). Respecto a la carne de bovino, su producción durante el 2020 fue de 2.1 millones de toneladas, con una proyección de 1.98 millones para el 2021 (SIAP, 2021). Los cortes que más demandan tienen entre los consumidores son el bistec, la milanesa y la carne molida (Domínguez-Vara y MondragónAncelmo, 2018). Esta última se utiliza generalmente para la preparación de alimentos como picadillo, albóndigas y hamburguesas. Las hamburguesas elaboradas con carne de bovino son excelente fuente de proteínas de alto valor biológico, ácidos grasos esenciales, vitaminas y minerales. Su incremento de producción en establecimientos de comida rápida, su conveniencia y bajo precio la han posicionado como uno de los productos cárnicos procesados más populares (Selani et al., 2016). Además, la hamburguesa se ha convertido en un atractivo principal en supermercados, distribuidores de comida rápida y restaurantes alrededor del mundo. Sin embargo, la hamburguesa es un producto cárnico procesado y como tal su consumo se ha asociado a enfermedades cardiovasculares y cáncer colorectal, debido principalmente a su contenido de grasas saturadas, colesterol y sal (do Prado et al., 2019). Ante estas situaciones, los consumidores se han vuelto más conscientes respecto a una dieta saludable, demandando productos con un perfil de lípidos 
mejorado, reducidos en colesterol y sodio; o formulados con ingredientes nóveles que tienen un probado efecto benéfico en la salud (do Prado et al., 2019). Además, buscan productos con etiqueta limpia; es decir, aquellos que tienen una lista de ingredientes corta, simple, sin connotaciones químicas, con ingredientes reconocibles y usualmente encontrados en una cocina (Asioli et al., 2017).

La adición de ingredientes no tradicionales como aceites vegetales, fibra o compuestos bioactivos ha resultado una estrategia exitosa para disminuir el contenido de grasas saturadas y mejorar el perfil nutricional en productos como las hamburguesas (Botez et al., 2017)2017. Entre los ingredientes con potencial para desarrollar productos cárnicos con una connotación más saludable se encuentran la harina de linaza, la pulpa de mango y la ciruela deshidratada. La harina de linaza es una excelente fuente de ácidos grasos omega-3, destacando el ácido linolénico con un $57 \%$ del total de los ácidos grasos, compuesto conocido por reducir niveles de colesterol y brindar beneficios a la salud (Bilek y Turhan, 2009). Sin embargo, no ha sido posible incorporar más del $3 \%$ en la formulación en productos cárnicos debido a un detrimento en las propiedades fisicoquímicas y sensoriales (Valenzuela-Melendres et al., 2018). Por otra parte, el mango y la ciruela deshidratada son fuente de antioxidantes como compuestos fenólicos, carotenoides y vitamina C, además de fibra dietética (Jarvis et al., 2015; Torres-León et al., 2016), y su incorporación en productos cárnicos reducen la oxidación lipídica y la cuenta total de microorganismos durante su vida de anaquel (Nuñez de González et al., 2008; Kadakadiyavar et al., 2017), además de mejorar las propiedades sensoriales, sin un efecto marcado en las características fisicoquímicas (Yıldız-Turp y Serdaroglu, 2010).

Se ha reportado la adición de estos ingredientes en carnes procesadas para mejorar sus propiedades nutricionales y tecnológicas. Por ejemplo, se ha incorporado harina de linaza como ingrediente en hamburguesas, obteniendo un producto con un mejor perfil de lípidos, pero un problema persistente es el detrimento en sus propiedades sensoriales y una mayor susceptibilidad al deterioro de los ácidos grasos (Valenzuela-Melendres et al., 2018). Por otra parte, diversos estudios han demostrado que la pulpa de mango es rica en compuestos antioxidantes que pueden ser incorporados para superar el problema de la oxidación de ácidos grasos polinsaturados, además de que pueden contribuir con mejores propiedades sensoriales en los productos cárnicos (Bhat et al., 2017). Por otro lado, la adición de la ciruela deshidratada en formulaciones cárnicas ha sido reportada por diversos investigadores, destacando su efecto positivo en parámetros sensoriales como la textura, jugosidad y sabor (Jarvis et al., 2015). Por todo lo anterior, es posible hipotetizar que tanto la pulpa de mango como la ciruela deshidratada pueden ser utilizadas para reducir el impacto negativo que la harina de linaza ejerce en las formulaciones cárnicas, buscando la combinación óptima entre los tres ingredientes que resulte en un producto con un contenido de ácidos grasos poliinsaturados mayor al de una hamburguesa tradicional, pero con el menor impacto en las propiedades fisicoquímicas y sensoriales.

Determinar los efectos e interacciones entre múltiples ingredientes sobre la calidad de un producto cárnico es complicado si no se utiliza la estrategia metodológica adecuada (Botez et al., 2017)2017. La metodología de superficie de respuesta (MSR) es una técnica matemática que permite optimizar la incorporación de nuevos ingredientes y ha sido utilizada con éxito en el desarrollo de nuevos productos cárnicos (Keenan et al., 2014; Valenzuela-Melendres et al., 2014). El objetivo del presente trabajo fue estudiar los efectos de harina de linaza, pulpa de mango y ciruela deshidratada sobre la calidad de hamburguesas de carne de bovino, y optimizar por MSR la incorporación de estos ingredientes en su formulación para generar un producto con mejor perfil nutricional, pero sin demérito en sus propiedades fisicoquímicas y sensoriales.

\section{MATERIALES Y MÉTODOS Diseño experimental}

Se estudiaron los efectos de harina de linaza $(\mathrm{HL})$, pulpa de mango (PM) y ciruela deshidratada (CD) sobre la calidad de hamburguesas de carne de bovino mediante el uso de la MSR, utilizando para ello un diseño de mezclas simplex centroide. Los niveles de los factores fueron: $\mathrm{HL}(0$ a $20 \%)$, PM (0 a $20 \%$ ) y CD (0 a $20 \%$ ) y con ellos se generaron 14 tratamientos o corridas experimentales (Tabla 1). Los tratamientos con los límites máximos de incorporación de cada ingrediente y el correspondiente a la mezcla de partes iguales (punto central del diseño), fueron repetidos durante el experimento para estimar el error experimental y la falta de ajuste de los modelos de regresión resultante. Las mediciones realizadas fueron: color instrumental, $\mathrm{pH}$, rendimiento de cocción $(R C)$, reducción del diámetro $(R D)$, análisis de perfil de textura (APT) y análisis sensorial (AS). Se realizaron dos repeticiones del experimento completo y en cada repetición se analizaron tres muestras por tratamiento para la evaluación de la calidad para un total de 6 muestras por tratamiento.

\section{Ingredientes utilizados, materiales y elaboración del producto}

La harina de linaza (Bob's Red Mill, Oregon, USA), pulpa de mango y ciruela deshidratada (Sunsweet Growers Inc., Yuba, CA, USA), fueron adquiridos de supermercados locales y usados como ingredientes para formular las hamburguesas. La carne de bovino magra (Semimembranoso, $72 \%$ humedad, $6 \%$ lípidos, $20 \%$ proteína y $2 \%$ ceniza), pH 5.82 fue adquirida con un distribuidor directo de carne local y utilizada el mismo día de su compra. La carne fue molida en un equipo Hobart (modelo 4152, Troy, Ohio, USA) a través de un plato con orificios de $4.7 \mathrm{~mm}$. La pulpa de mango se obtuvo de frutos de la variedad Haden en estado de madurez de consumo, sin deformaciones ni daños en la cutícula. La harina de linaza fue directamente añadida en 
Tabla 1. Composición de las mezclas en hamburguesas de carne de bovino formuladas con harina de linaza, pulpa de mango y ciruela deshidratada en un diseño de mezclas simplex centroide de tres componentes.

Table 1. Composition of mixtures in beef patties formulated with flaxseed flour, mango pulp and dried plum in a simplex centroid three-component mixture design.

\begin{tabular}{|c|c|c|c|c|c|c|}
\hline \multirow[b]{2}{*}{ Tratamiento } & \multicolumn{3}{|c|}{ Valores codificados } & \multicolumn{3}{|c|}{ Valores reales (\%) } \\
\hline & HL & PM & $C D$ & HL & PM & CD \\
\hline 1 & 1 & 0 & 0 & 20 & 0 & 0 \\
\hline 2 & 0 & 1 & 0 & 0 & 20 & 0 \\
\hline 3 & 0 & 0 & 1 & 0 & 0 & 20 \\
\hline 4 & 0 & 0.5 & 0.5 & 0 & 10 & 10 \\
\hline 5 & 0.5 & 0 & 0.5 & 10 & 0 & 10 \\
\hline 6 & 0.5 & 0.5 & 0 & 10 & 10 & 0 \\
\hline 7 & 0.33 & 0.33 & 0.33 & 6.67 & 6.67 & 6.67 \\
\hline 8 & 0.67 & 0.17 & 0.17 & 13.32 & 3.34 & 3.34 \\
\hline 9 & 0.17 & 0.67 & 0.17 & 3.34 & 13.32 & 3.34 \\
\hline 10 & 0.17 & 0.17 & 0.67 & 3.34 & 3.34 & 13.32 \\
\hline 11 & 1 & 0 & 0 & 20 & 0 & 0 \\
\hline 12 & 0 & 1 & 0 & 0 & 20 & 0 \\
\hline 13 & 0 & 0 & 1 & 0 & 0 & 20 \\
\hline 14 & 0.33 & 0.33 & 0.33 & 6.67 & 6.67 & 6.67 \\
\hline
\end{tabular}

$\mathrm{HL}=$ harina de linaza, $\mathrm{PM}=$ pulpa de mango, $\mathrm{CD}=$ ciruela deshidratada.

Proporción de $\mathrm{HL}+\mathrm{PM}+\mathrm{CD}=1$.

las hamburguesas. Se utilizó un procesador de alimentos (NutriBullet NUBPT0101, Los Angeles, CA, USA) para moler en forma separada la pulpa de mango y ciruela deshidratada hasta obtener un puré. Lotes de $1 \mathrm{~kg}$ por tratamiento fueron usados para la preparación de las hamburguesas. La carne, la harina de linaza, la pulpa de mango y la ciruela deshidratada fueron mezclados de acuerdo a los porcentajes especificados en las corridas experimentales de la Tabla 1. Los ingredientes en cada lote fueron homogenizados manualmente por 10 min y las hamburguesas fueron formadas manualmente en muestras de $9 \mathrm{~cm} \times 1 \mathrm{~cm}$ de grosor para obtener $60 \mathrm{~g}$ por unidad. Las hamburguesas fueron cocinadas en un sartén eléctrico (Cook Master Oster, Model 3222-3, Mississauga, Ontario, Canadá) durante 1 min por cada lado y después $15 \mathrm{~s}$ por lado hasta alcanzar $72{ }^{\circ} \mathrm{C}$ medidos por un termopar en el centro geométrico de la hamburguesa.

\section{Color instrumental, pH, rendimiento de cocción y encogi- miento al cocinar}

El color fue evaluado manteniendo todos los tratamientos a temperatura ambiente $\left(25 \pm 2{ }^{\circ} \mathrm{C}\right)$ por 5 min antes de su medición utilizando un colorímetro (Chroma meter CR-400, Konica Minolta Sensing, Inc., Osaka, Japan) con iluminante $\mathrm{D} 65$, observador de $10^{\circ}$ y apertura de $11 \mathrm{~mm}$ del instrumento para iluminación y $8 \mathrm{~mm}$ para medición. Las coordenadas determinadas fueron: luminosidad $\left(L^{*}\right.$, donde $0=$ negro y $100=$ blanco $)$, matiz verde-rojo $\left(a^{*}\right.$, donde $-60=$ verde $\mathrm{y}+60=$ rojo $)$ y matiz azul-amarillo $\left(b^{*}\right.$, donde $-60=$ azul $y+60=$ amarillo). Se tomaron nueve repeticiones de cada muestra, siguiendo las guías para la evaluación colorimétrica de carnes.

El $\mathrm{pH}$ fue medido directamente en hamburguesas cocinadas con un potenciómetro portátil (Hanna, Model HI 98140, Woonsocket, RI, USA) equipado con una combinación electrodos de $\mathrm{pH}$ tipo punción.

Las hamburguesas fueron pesadas antes y después de ser cocinadas utilizando una balanza de precisión (L-EQ Torrey, Monterrey, México), después se enfriaron a $25^{\circ} \mathrm{C}$ y secaron con una toalla de papel para retirar el agua y la grasa liberadas durante el cocinado. El rendimiento de cocción (RC) fue calculado por medio de una fórmula (Ec. 1):

$$
R C=\frac{m 2}{m 1} * 100
$$

Donde $m 1$ es el peso ( $\mathrm{g}$ ) de la hamburguesa antes de cocción y $m 2$ es el peso $(\mathrm{g})$ después de cocción (Szerman et al., 2019). Por otro lado, la reducción del diámetro (RD) en las hamburguesas por el proceso de cocción fue calculado por medio de la fórmula (EC. 2):

$$
R D=\frac{D 1-D 2}{D 1} * 100
$$

Donde $D 1$ es el diámetro $(\mathrm{cm})$ de la hamburguesa cruda y $D 2$ es el diámetro $(\mathrm{cm})$ de la hamburguesa cocinada. 


\section{Análisis de perfil de textura}

El análisis de perfil de textura se realizó en muestras de hamburguesas cocinadas, a $4 \pm 1{ }^{\circ} \mathrm{C}$ con un analizador de textura TA-XT2 (Stable Micro Systems, Surrey, UK) siguiendo los métodos para la medición de textura de productos cárnicos (Claus, 1995). Muestras cubicas $(1 \times 1 \times 1 \mathrm{~cm})$ fueron cortadas de las hamburguesas cocinadas y sometidas a pruebas de compresión de dos ciclos. Las muestras fueron comprimidas hasta $50 \%$ de su altura original con una sonda cilíndrica de $75 \mathrm{~mm}$ de diámetro unida a una celda de compresión de 50 $\mathrm{kg}$ con una velocidad de cabezal de $1 \mathrm{~mm} / \mathrm{s}$. Los parámetros de perfil de textura fueron determinados de acuerdo a Bourne (1978), e interpretados de la siguiente manera: dureza (N) como la fuerza máxima requerida para comprimir la muestra; cohesividad como la extensión en la cual la muestra puede ser deformada antes de su ruptura (A2/A1), donde A1 es la energía total requerida para la primer compresión y $A 2$ es la energía total requerida para la segunda compresión; elasticidad $(\mathrm{cm})$ como la habilidad de la muestras para recuperar su forma original después de que la fuerza de deformación es removida; masticabilidad $(\mathrm{N} / \mathrm{cm})$ como el trabajo necesario para masticar la muestra y posteriormente engullirla (dureza $\times$ cohesividad $\times$ elasticidad).

\section{Análisis sensorial}

La evaluación sensorial se realizó por quince panelistas, 8 hombres y 7 mujeres, con experiencia previa en análisis sensorial y consumidores regulares de hamburguesas. El análisis sensorial se realizó en dos días diferentes, en los que todos los panelistas evaluaron 7 muestras por día, seleccionadas al azar. El panel estuvo integrado por personal y estudiantes de la Coordinación de Tecnología de Alimentos de Origen Animal de la institución. La evaluación sensorial se realizó en un cuarto dividido en cabinas individuales y ambiente controlado $\left(23 \pm 1{ }^{\circ} \mathrm{C}, 55 \pm 5 \% \mathrm{HR}\right)$. Previo al análisis, los panelistas obtuvieron un entrenamiento donde se explicó el objetivo del proyecto, se explicaron los descriptores a calificar y la escala de evaluación. Durante la evaluación sensorial, cuatro hamburguesas de cada formulación fueron cocinadas como se describió previamente, cubiertas y mantenidas a $60{ }^{\circ} \mathrm{C}$ en un horno hasta su evaluación por 4-7 min. Las hamburguesas fueron cortadas en cuatro partes iguales y servidas inmediatamente. Cada muestra fue codificada con números de tres dígitos aleatoriamente seleccionados. Los panelistas fueron instruidos para limpiar sus paladares entre cada muestra utilizando agua y galletas sin sal. Cada panelista evaluó todas las formulaciones en un orden aleatorio y se les pidió evaluar en una escala de 0 a 10 los siguientes atributos: sabor ( $0=$ nada intenso/10 = muy intenso), textura $(0=$ dura $/ 10=$ blanda) y jugosidad $(0=$ seca $/ 10=$ jugosa $)$.

\section{Análisis estadístico de los datos}

Para el análisis estadístico, se utilizó la MSR. Primeramente, cada variable respuesta fue ajustada a un modelo cúbico especial (Ec. 3) para modelar los datos obtenidos en el experimento. $y=\beta_{1} x_{1}+\beta_{2} x_{2}+\beta_{3} x_{3}+\beta_{12} x_{1} x_{2}+\beta_{13} x_{1} x_{3}+\beta_{23} x_{2} x_{3}+\beta_{123} x_{1} x_{2} x_{3}$ (EC. 3)

donde $y$ es la variable de respuesta (color, $\mathrm{pH}, \mathrm{RC}, \mathrm{RD}$, APT y AS), $\beta$ es el coeficiente de regresión y $x$ es la proporción de los componentes ( $\mathrm{HL}, \mathrm{PM}$ y $\mathrm{CD}$ ) en la formulación. Para el análisis de resultados se utilizó el software Design Expert 7.0.0 (Stat-Ease, Inc., Minneapolis, MN, USA). Para cada variable respuesta, se realizó un análisis de varianza para estimar las significancias $(p<0.05)$ de los factores estudiados. Se estimaron los coeficientes de regresión, el coeficiente de determinación $\left(R^{2}\right)$ y falta de ajuste de los modelos resultantes.

Con los modelos predictivos seleccionados, se llevó a cabo la optimización de las respuestas, mediante la técnica de optimización numérica del software Design Expert para la optimización simultánea de múltiples respuestas. Para el presente estudio, la formulación óptima de hamburguesa de carne de bovino se predijo maximizando la incorporación de HL, PM y CD. Se eligieron los objetivos deseados para cada variable de respuesta. La formulación óptima se usó para estimar los valores predichos de las variables de respuesta utilizando las ecuaciones de predicción derivadas de la MSR. Para la validación de los modelos, se preparó un lote de hamburguesas con los niveles óptimos de los ingredientes, se evaluaron experimentalmente las propiedades fisicoquímicas y sensoriales de acuerdo las metodologías descritas anteriormente, y se compararon estadísticamente $(p<0.05)$ con los valores predichos por los modelos. Finalmente, se realizó una comparación entre la formulación optimizada y una formulación típica de hamburguesas con 20 \% de grasa de bovino en términos de composición proximal y perfil de ácidos grasos. La composición proximal (humedad, grasa, proteína y ceniza), se realizó siguiendo las técnicas descritas por la AOAC (1990), mientras que el perfil de ácidos grasos se realizó de acuerdo a la técnica especificada por ValenzuelaMelendres et al. (2018).

\section{RESULTADOS Y DISCUSIÓN Propiedades fisicoquímicas}

La Tabla 2 muestra los valores experimentales resultantes para todas las propiedades fisicoquímicas. En la Tabla 3 se muestran los modelos de regresión obtenidos para predecir el color instrumental $\left(L^{*}, a^{*}, b^{*}\right), \mathrm{pH}$, rendimiento de cocción $(\mathrm{RC})$, reducción de diámetro $(\mathrm{RD})$ y análisis de perfil de textura de hamburguesas de carne de bovino, en función de la adición de harina de linaza $(\mathrm{HL})$, pulpa de mango (PM) y ciruela deshidratada (CD). Se muestra también el análisis de varianza, la falta de ajuste y el coeficiente de determinación $\left(R^{2}\right)$ de los modelos de regresión para cada variable de respuesta estudiada. Un signo positivo en el modelo de regresión indica que a medida que se adiciona el ingrediente, la variable de respuesta también lo hace. Un signo negativo en el modelo indica que a medida que se adiciona el ingrediente, la variable de respuesta disminuye. Las Figuras 1-2 muestran los efectos y tendencias de las variables de proceso para cada propiedad fisicoquímica del producto. 
Tabla 2. Propiedades fisicoquímicas y sensoriales de hamburguesas de carne de bovino formuladas con harina de linaza, pulpa de mango y ciruela deshidratada.

Table 2. Physicochemical and sensory properties of beef patties formulated with flaxseed flour, mango pulp and dried plum.

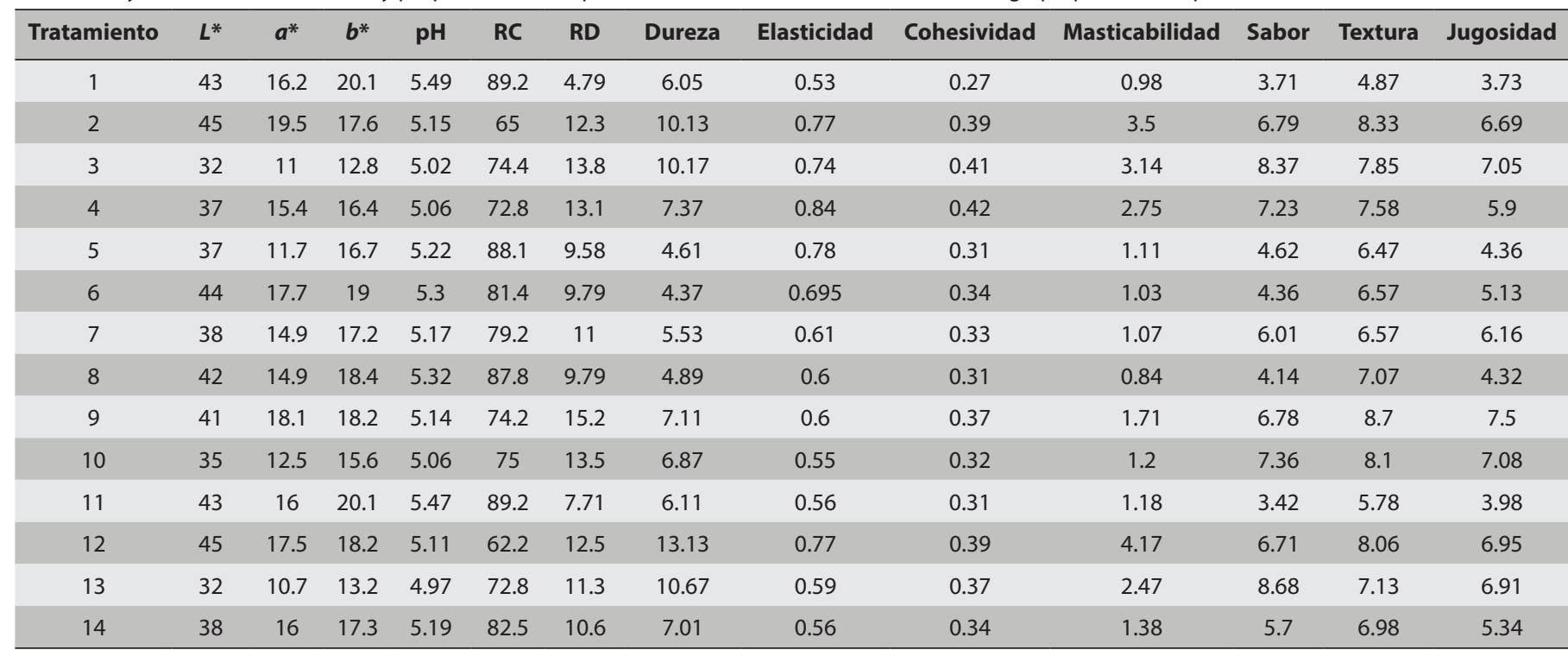

$\mathrm{RC}=$ rendimiento por cocción (\%), $\mathrm{RD}=$ reducción de diámetro $(\%)$, dureza $(\mathrm{N})$, elasticidad $(\mathrm{cm})$, cohesividad (adimensional), masticabilidad $(\mathrm{N} / \mathrm{cm})$, sabor $(0$ $=$ nada intenso $/ 10=$ muy intenso), textura $(0=$ dura $/ 10=$ blanda) $y$ jugosidad $(0=$ seca $/ 10=$ jugosa $)$.
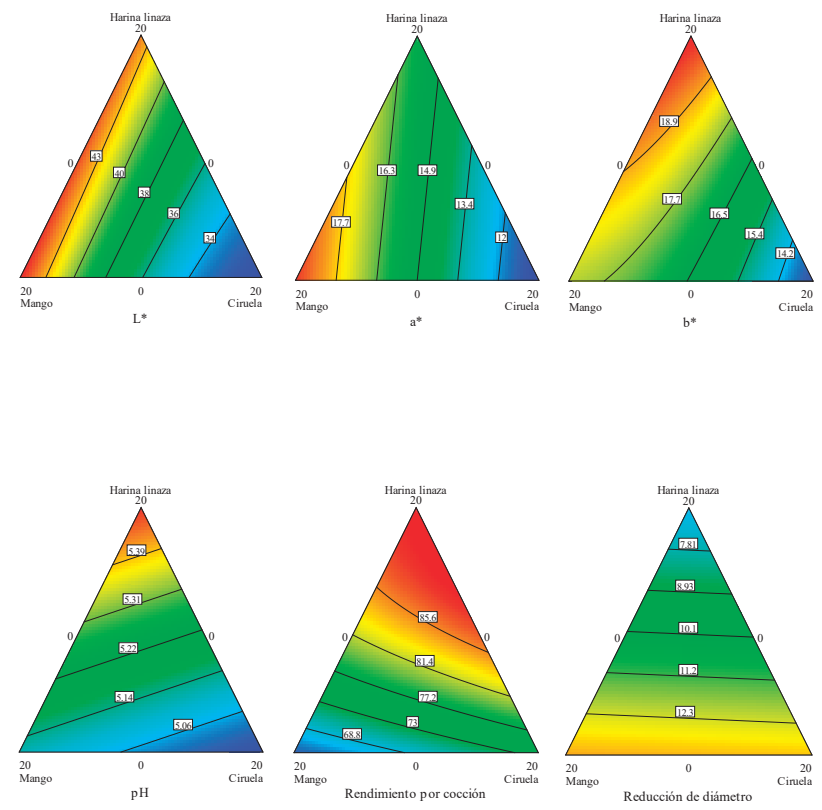

Figura 1. Gráfico de contorno de diseño de mezclas para color instrumental: $L^{*}$ (luminosidad), $a^{*}$ (matiz verde-rojo) y c) y $b^{*}$ (matiz azul-amarillo), $\mathrm{pH}$, rendimiento por cocción y reducción de diámetro en hamburguesas de carne de bovino adicionadas con HL, PM y CD.

Figure 1. Mixture design contour plot for instrumental color: $L^{*}$ (lightness), $a^{*}$ (green-red axe) and $b^{*}$ (blue-yellow axe), $\mathrm{pH}$, cooking yield and diameter reduction, in added beef patties added with FS, MP and DP.
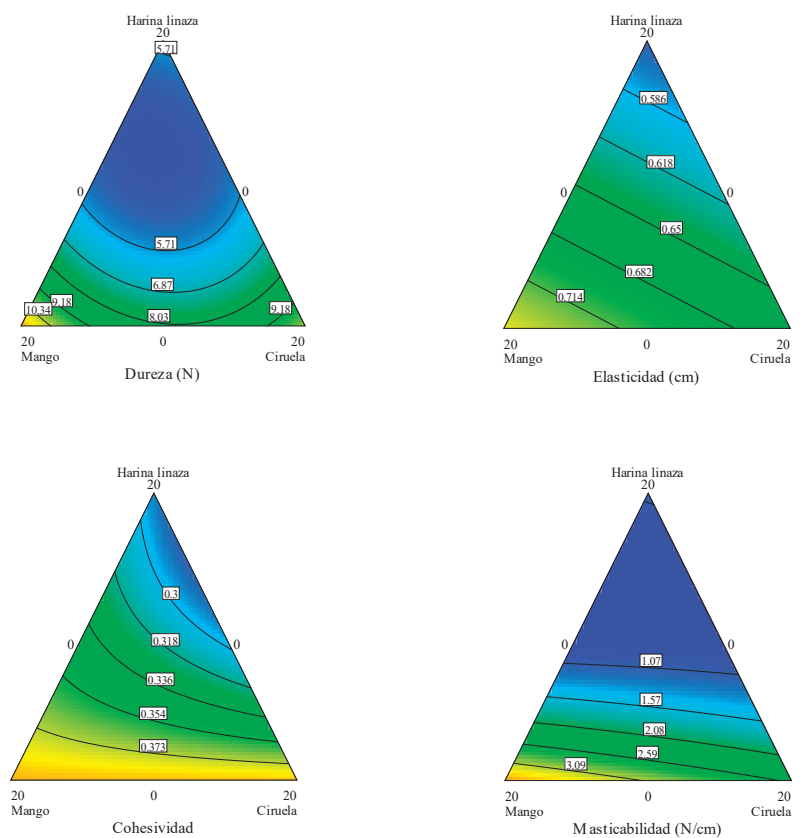

Figura 2. Gráfico de contorno de diseño de mezclas para perfil de textura: dureza, elasticidad, cohesividad y masticabilidad de hamburguesas de carne de bovino adicionadas con $\mathrm{HL}, \mathrm{PM}$ y CD.

Figure 2. Mixture design contour plot for texture profile analysis: hardness, elasticity, cohesiveness and chewiness of beef patties added with FS, MP and DP. 


\section{Color instrumental}

Los modelos de regresión para los valores $L^{*}, a^{*} y$ $b^{*}$ fueron significativos $(p<0.05)$, tuvieron un coeficiente de determinación mayor a 0.92 y no presentaron falta de ajuste $(p>0.05)$, elementos suficientes que indican que son adecuados para predecir la variable de respuesta. La Figura 1 muestra la gráfica de contorno para los valores $L^{*} a^{*}$ y $b^{*}$ en función de la adición de HL, PM y CD. La Tabla 2 muestra los valores del color instrumental para todos los tratamientos evaluados, donde los promedios resultantes para $L^{*}, a^{*}$ y $b^{*}$ fueron $39.04,15.37$ y 16.82 , respectivamente. El color de los productos cárnicos depende de varios factores que incluyen la cantidad y estado químico de la mioglobina, las características fisicoquímicas de la carne, y el tipo y cantidad de ingredientes no cárnicos en la formulación del producto (Cofrades et al., 2004).

De acuerdo a los modelos de regresión, todos los parámetros del color instrumental aumentaron $(p<0.05)$ por la incorporación de PM y HL, y disminuyeron $(p<0.05)$ al incorporar CD en la formulación cárnica. El efecto que tiene la adición de PM en el incremento del color del producto puede deberse a varias razones; por un lado, la alta humedad de la PM provoca un mayor reflejo de la luz sobre la superficie del alimento. Por otro lado, la PM es rica en compuestos antioxidantes, principalmente carotenoides, que retardan la oxidación de la mioglobina de la carne, responsable del color rojo característico de este producto. Wongkaew et al. (2020) adicionaron pectina de cáscara de mango a salchichas de cerdo hasta un nivel del $15 \%$ y reportaron un incremento del color en el producto atribuido a los compuestos carotenoides presentes en la cáscara de mango. Resultados similares fueron también reportados por Qiu y Chin (2021), quienes encontraron que los carotenoides presentes en el tomate mejoraron el color de hamburguesas de carne de puerco.

Por el contrario, el efecto de la CD sobre la disminución del color puede ser atribuido al color oscuro propio del fruto; este comportamiento es similar a lo reportado por Reyes-Padilla et al. (2018), donde la incorporación de $5 \%$ $\mathrm{CD}$ en bolonia generó los valores $L^{*}, a^{*}$ y $b^{*}$ más bajos. De igual manera, Nuñez de Gonzalez et al. (2008), reportaron una disminución en los valores $L^{*}, a^{*}$ y $b^{*}$ en carne de bovino cuando la cantidad de CD en la formulación se incrementó de $2.5 \%$ a $5 \%$.

\section{pH, rendimiento por cocción y reducción de diámetro}

$\mathrm{El} \mathrm{pH}, \mathrm{RC}$ y RD de las hamburguesas de carne de bovino estuvieron en los rangos de 4.97 - 5.49, 62.2 - 89.17 y 4.79 - 15.20, respectivamente (Tabla 2). Todos los modelos de regresión para $\mathrm{pH}, \mathrm{RC}$ y $\mathrm{RD}$ fueron significativos $(p<0.05)$, con una $\mathrm{R}^{2}$ aceptable y con una falta de ajuste mayor a 0.05 (Tabla 3), por lo cual son adecuados para predecir la variable de respuesta. La Figura 1 muestra los efectos y tendencias de las variables de proceso para el $\mathrm{pH}, \mathrm{RC}$ y $\mathrm{RD}$, respectivamente. El pH es un parámetro de calidad muy importante en la carne, ya que puede estar relacionado directa o indirectamente con otros parámetros de calidad como la capacidad de retención

Tabla 3. Modelos de regresión de los parámetros de calidad en el diseño de mezclas.

Table 3. Regression models of the quality parameters in the mixture design.

\begin{tabular}{|c|c|c|c|c|c|}
\hline VR & $\begin{array}{l}\text { a Promedio } \\
\quad \pm D E\end{array}$ & $\mathbf{R}^{2}$ & FA & Prob $<p$ & Modelo de regresión \\
\hline \multicolumn{6}{|c|}{ Color, pH y propiedades de cocción } \\
\hline$L^{*}$ & $39.40 \pm 4.41$ & 0.99 & 0.07 & $<0.0001$ & $5.43 \mathrm{HL}+7.13 \mathrm{PM}-12.55 \mathrm{CD}-4.44 \mathrm{HL}^{*} \mathrm{CD}-8.18 \mathrm{PM}{ }^{*} \mathrm{CD}$ \\
\hline$a^{*}$ & $15.10 \pm 2.65$ & 0.92 & 0.51 & $<0.0001$ & $0.88 \mathrm{HL}+5.89 \mathrm{PM}-6.77 \mathrm{CD}$ \\
\hline$b^{*}$ & $17.20 \pm 2.10$ & 0.99 & 0.65 & $<0.0001$ & $4.27 \mathrm{HL}+1.51 \mathrm{PM}-5.78 \mathrm{CD}+3.90 \mathrm{PM}^{*} \mathrm{CD}$ \\
\hline Rendimiento (\%) & $78.10 \pm 8.40$ & 0.96 & 0.27 & $<0.0001$ & 21.47 HL $-17.86 \mathrm{PM}-3.61 \mathrm{CD}+19.89 \mathrm{HL}^{*} \mathrm{PM}+22.51 \mathrm{HL}^{*} \mathrm{CD}$ \\
\hline Encogimiento (\%) & $11.10 \pm 2.60$ & 0.76 & 0.47 & 0.0004 & $-6.56 \mathrm{HL}+3.50 \mathrm{PM}+3.06 \mathrm{CD}$ \\
\hline $\mathrm{pH}$ & $5.19 \pm 0.15$ & 0.98 & 0.83 & $<0.0001$ & $0.42 \mathrm{HL}-0.10 \mathrm{PM}-0.31 \mathrm{CD}$ \\
\hline \multicolumn{6}{|c|}{ Análisis de perfil de textura } \\
\hline Dureza (N) & $7.43 \pm 2.53$ & 0.89 & 0.61 & 0.0012 & $\begin{aligned}-4.95 \mathrm{HL}+3.20 \mathrm{PM}+ & 1.75 \mathrm{CD}-14.01 \mathrm{HL}{ }^{*} \mathrm{PM}-10.56 \mathrm{HL}^{*} \mathrm{CD} \\
& -11.09 \mathrm{PM}^{*} \mathrm{CD}\end{aligned}$ \\
\hline Elasticidad (cm) & $0.66 \pm 0.09$ & 0.34 & 0.12 & 0.103 & $-0.15 \mathrm{HL}+0.14 \mathrm{PM}+0.02 \mathrm{CD}$ \\
\hline Cohesividad & $0.35 \pm 0.04$ & 0.83 & 0.56 & 0.0003 & $-0.10 \mathrm{HL}+0.06 \mathrm{PM}+0.04 \mathrm{CD}-0.18 \mathrm{HL}{ }^{*} \mathrm{CD}$ \\
\hline $\begin{array}{l}\text { Masticabilidad (N/ } \\
\mathrm{cm})\end{array}$ & $1.89 \pm 1.06$ & 0.89 & 0.28 & 0.0002 & $-2.25 \mathrm{HL}+1.81 \mathrm{PM}+0.44 \mathrm{CD}-6.37 \mathrm{HL}^{*} \mathrm{PM}-4.15 \mathrm{HL}^{*} \mathrm{CD}$ \\
\hline \multicolumn{6}{|c|}{ Propiedades sensoriales } \\
\hline Sabor & $5.99 \pm 1.65$ & 0.96 & 0.06 & $<0.0001$ & $-4.39 \mathrm{HL}+1.00 \mathrm{PM}+3.39 \mathrm{CD}-4.31 \mathrm{HL}{ }^{*} \mathrm{CD}$ \\
\hline Textura & $7.15 \pm 1.02$ & 0.76 & 0.27 & 0.0004 & $-2.49 \mathrm{HL}+1.77 \mathrm{PM}+0.72 \mathrm{CD}$ \\
\hline Jugosidad & $5.72 \pm 1.38$ & 0.84 & 0.14 & $<0.0001$ & $-3.66 \mathrm{HL}+1.91 \mathrm{PM}+1.75 \mathrm{CD}$ \\
\hline
\end{tabular}

$\mathrm{VR}=$ variable respuesta, $\mathrm{FA}=$ falta de ajuste, $0<\mathrm{R}^{2}<1$ (cercano a I más significativo), a Promedio de 6 determinaciones $\pm \mathrm{DE}$ (desviación estándar), HL (harina de linaza), PM (pulpa de mango), CD (ciruela deshidratada), * $p<0.05$ 
de agua, propiedades organolépticas y calidad microbiológica (Toldrá, 2003). De acuerdo a los modelos de regresión, la incorporación de PM y CD aumentó $(p<0.05)$ la acidez en las hamburguesas, mientras que la adición de HL la disminuyó. Los efectos de estos ingredientes sobre el $\mathrm{pH}$ del producto pueden atribuirse al $\mathrm{pH}$ propio de cada uno de ellos; por un lado, la PM y CD poseen un pH de 3.45 y 3.82, respectivamente, debido principalmente a la alta cantidad de ácidos orgánicos como cítrico y málico (Jarvis et al., 2015; Maldonado-Celis et al., 2019), razón por la cual al ser incorporados en el alimento le confieren un $\mathrm{pH}$ más ácido, mientras que la $\mathrm{HL}(\mathrm{pH}=6.42)$ le confiere al alimento un $\mathrm{pH}$ más alcalino. Yıldız-Turp y Serdaroglu (2010), reportaron que se observa una disminución de $\mathrm{pH}$ aumentando las proporciones de $\mathrm{CD}$ en hamburguesas de carne de bovino; de manera similar se ha reportado una disminución de $\mathrm{pH}$ en milanesas de pollo debido a un aumento en la adición de polvo de cáscara de mango (Bhat et al., 2017). Los efectos anteriormente explicados se pueden apreciar en el gráfico de contorno de diseño de mezclas de la Figura 1, donde se observa que una mayor concentración de HL en la formulación del producto provoca una disminución de la acidez mientras que una mayor concentración de PM o CD ocasiona un aumento de la misma.

El RC y RD son parámetros tecnológicos importantes en productos cárnicos ya que determinan las características finales del alimento; éstos son función de la pérdida de agua y grasa del alimento por efecto de la temperatura de cocinado; a mayor pérdida de agua y grasa durante el cocimiento, menor será el RC y mayor la RD (Yıldız-Turp y Serdaroglu, 2010). El pH de cada ingrediente es uno de los aspectos importantes que hay que tomar en cuenta, pues éste influye directamente en la capacidad de retención de agua del producto. El bajo $\mathrm{pH}$ de la PM (pH = 3.45) y CD (pH = 3.82) tiene un efecto directo sobre el RC y RD, ya que mantiene a las proteínas miofibrilares de la carne cercanas a su punto isoeléctrico $(\mathrm{pl}=5.1-5.2)$, que es donde la capacidad de retención de agua es mínima (Toldrá, 2003). Un efecto contrario se observa por la adición de $\mathrm{HL}(\mathrm{pH}=6.42)$. La PM produce un menor RC y una mayor $\mathrm{RD}$ debido a que al ser un ingrediente fresco (a diferencia de la $C D$ ) éste aporta una alta cantidad de agua al producto cárnico y promueve la pérdida de peso durante el proceso de cocinado. En Figura 1 se observa gráficamente como un mayor porcentaje de HL ocasiona un aumento del RC y una disminución de RD, mientras que un mayor porcentaje de PM o CD provoca una disminución del RC y un aumento de RD.

\section{Análisis de perfil de textura}

Los rangos registrados de dureza $(\mathrm{N})$, elasticidad $(\mathrm{cm})$ y masticabilidad $(\mathrm{N} / \mathrm{cm})$ oscilaron entre $4.4-13.1,0.53-$ 0.84 y $0.8-4.1$, respectivamente (Tabla 2). En relación a la cohesividad, los valores fueron muy uniformes entre todos los tratamientos evaluados presentando un rango de $0.3-$ 0.4 . Todos los modelos de regresión para el perfil de textura fueron significativos $(p<0.05)$, con una $\mathrm{R}^{2}$ aceptable y con una falta de ajuste mayor a 0.05 (Tabla 3), por lo cual los modelos son adecuados para predecir la variable de respuesta.
La representación gráfica de los modelos de regresión para el perfil de textura obtenidos de la matriz de diseño de mezclas se presenta en la Figura 2.

La textura de un producto cárnico es una de las características de calidad más importantes que determina su aceptación por parte del consumidor (Braña-Varela et al., 2011). De acuerdo a los modelos obtenidos, la incorporación de HL disminuyó $(p<0.05)$ todos los valores de textura, lo que evidencia el detrimento provocado por este ingrediente sobre la calidad tecnológica del producto. El efecto negativo de este ingrediente en las propiedades de textura de hamburguesas de carne de bovino ha sido reportado también por Valenzuela-Melendres et al. (2014), quienes mencionan que la adición de más del $3 \%$ de HL en la formulación causa un detrimento marcado en la textura del producto. Por otra parte, la PM y CD aumentaron los valores del perfil de textura $(p<0.05)$ en comparación a lo observado por la incorporación de HL. Ambos ingredientes presentaron un efecto muy similar en todos los parámetros de textura. El aumento de la dureza por la incorporación de PM y CD puede ser atribuido a los carbohidratos estructurales de estos frutos como agar, pectinas, almidón o carrageninas que pueden actuar como espesantes y gelificantes para mantener un producto más firme (Maldonado-Celis et al., 2019; Serna-Cock et al., 2016).

\section{Análisis sensorial}

La evaluación sensorial del sabor, textura y jugosidad oscilaron en los rangos de $3.42-8.68$, $4.87-8.77$ y $3.73-7.50$, respectivamente (Tabla 2). Todos los modelos de regresión para los atributos sensoriales fueron significativos $(p<0.05)$, con una $\mathrm{R}^{2}$ aceptable y con una falta de ajuste mayor a 0.05 (Tabla 3), por lo cual los modelos son adecuados para predecir la variable de respuesta. Un signo negativo en el modelo de regresión indica que a medida que se adiciona el ingrediente, la variable de respuesta disminuye; por el contrario, un signo positivo en el modelo indica que la variable de respuesta aumenta a medida que se incorpora el ingrediente.

Todas las propiedades sensoriales evaluadas aumentaron significativamente $(p<0.05)$ por la incorporación de $C D$ y $P M$ en la formulación cárnica, mientras que disminuyeron significativamente $(p<0.05)$ por la incorporación de $\mathrm{HL}$. Los efectos de PM, CD y $\mathrm{HL}$, se observan visualmente en los gráficos de contorno de la Figura 3, donde una mayor incorporación de PM y CD resulta en un aumento en la textura, jugosidad y sabor del producto, mientras que un efecto contrario se obtiene con la adición de HL. La harina de linaza es un ingrediente con un bajo contenido de humedad (7\%), y que cuando se incorpora a los productos cárnicos imparte una sensación de resequedad, además contiene una alta cantidad de aceites poliinsaturados propensos a la oxidación que impactan en la textura, sabor y jugosidad de los productos cárnicos (Valenzuela-Melendres et al., 2014). Por otro lado, los carbohidratos complejos como almidón y fibra en pulpa de mango pueden llegar hasta un $15 \%$ y 1.6 $\%$, respectivamente (Lebaka et al., 2021); estos componentes pueden actuar como espesantes y gelificantes al incorporar- 
se en la formulación cárnica, favoreciendo su textura. Por su parte, la ciruela deshidratada es rica en compuestos humectantes como el sorbitol cuyo contenido puede ser de hasta $21.7 \%$, según su origen (Gill et al., 2019), y que de manera natural disminuye la sensación de resequedad al masticar de formulaciones cárnicas que la contiene, favoreciendo la textura y jugosidad del producto. Además, la ciruela deshidratada imparte notas aromáticas y dulces a los productos cárnicos, lo que incrementa los valores del sabor en la evaluación sensorial (Nuñez de Gonzalez et al., 2009). Resultados similares a nuestro estudio fueron obtenidos por Yıldız-Turp y Serdaroglu (2010), quienes reportaron un incremento en la textura, jugosidad y sabor de hamburguesas de carne de bovino a las que se les incorporó hasta un $10 \%$ de puré de ciruela en la formulación.

\section{Optimización de hamburguesas de carne de bovino y validación de modelos}

Durante el proceso de optimización se establecen las condiciones que satisfacen simultáneamente los requerimientos de calidad que se deseen en el producto (Baugreet et al., 2017), siendo la función de deseabilidad uno de los enfoques más populares para lograr dicha optimización. En el presente estudio, se utilizó la herramienta de optimización numérica del software Desing Expert para estimar la combinación óptima de pulpa de mango, ciruela deshidratada y harina de linaza que maximizan las propiedades fisicoquímicas y sensoriales de hamburguesas de carne de bovino. La combinación óptima de estos ingredientes se logró cuando se alcanzó la máxima deseabilidad. En el proceso de optimización no se tomó en cuenta la propiedad de elasticidad del APT debido a que el modelo no fue significativo $(p=0.103)$ y presentó una $\mathrm{R}^{2}$ muy baja (0.34). La formulación óptima de hamburguesa de carne de bovino resultante incluyó $6 \%$ $\mathrm{HL}, 10.5 \%$ PM y $3.5 \% \mathrm{CD}$, con una deseabilidad de 0.506 . Los valores predichos por el modelo y los obtenidos experimentalmente para la formulación óptima de hamburguesas de carne de bovino se presentan en la Tabla 4. De acuerdo al análisis estadístico realizado, los valores predichos por el modelo y los obtenidos experimentalmente para la formulación óptima fueron similares $(p>0.05)$, por lo tanto, las ecuaciones de regresión obtenidas son adecuadas para estimar las propiedades fisicoquímicas y sensoriales de hamburguesas de carne de bovino adicionadas con pulpa de mango, ciruela deshidratada y harina de linaza.

\section{Composición proximal y perfil de ácidos grasos de la formulación óptima}

Se preparó un lote de hamburguesas con la formulación optimizada y se comparó con una hamburguesa típica con $20 \%$ de grasa en términos de composición proximal y perfil de ácidos grasos. Los resultados de esta comparación se muestran en la Tabla 5. En cuanto a la composición proximal, se observa que no hubo diferencias $(p>0.05)$ entre los tratamientos evaluados, respecto al contenido de proteínas
Tabla 4. Valores experimentales y valores predichos de hamburguesas de carne de bovino optimizadas en sus propiedades fisicoquímicas y sensoriales.

Table 4. Experimental and predicted values of beef patties optimized in their physicochemical and sensory properties.

\begin{tabular}{lccc}
\hline Respuesta & $\begin{array}{c}\text { Valor } \\
\text { predicho }\end{array}$ & IC 95\% & $\begin{array}{c}\text { Valor } \\
\text { experimental* }\end{array}$ \\
\hline Color, pHy propiedades de cocción & 41.1 & $40.8-41.5$ & 41.44 \\
\hline$L^{*}$ & 16.6 & $16.0-17.2$ & 16.00 \\
\hline$a^{*}$ & 18.1 & $17.9-18.2$ & 18.14 \\
\hline$b^{*}$ & 77.9 & $75.7-80.1$ & 78.33 \\
\hline $\begin{array}{l}\text { Rendimiento por } \\
\text { cocción (\%) }\end{array}$ & 11.3 & $10.4-12.3$ & 11.67 \\
\hline $\begin{array}{l}\text { Reducción de } \\
\text { diámetro (cm) }\end{array}$ & 5.20 & $5.19-5.21$ & 5.24 \\
\hline pH & & & \\
\hline Análisis de perfil de textura & & & \\
\hline Dureza (N) & 5.87 & $4.61-7.13$ & 7.03 \\
\hline Cohesividad & 0.35 & $0.34-0.37$ & 0.35 \\
\hline Masticabilidad (N/cm) & 1.47 & $0.99-1.94$ & 1.43 \\
\hline Propiedades sensoriales & & & \\
\hline Sabor & 5.80 & $5.54-6.06$ & 5.74 \\
\hline Textura & 7.36 & $6.97-7.74$ & 7.44 \\
\hline Jugosidad & 5.86 & $5.43-6.29$ & 5.55 \\
\hline
\end{tabular}

Promedio de 5 determinaciones.

y ceniza, oscilando éstos entre 15.96 a $17.04 \%$ y 1.48 a 1.55 $\%$, respectivamente. La humedad de la hamburguesa optimizada fue mayor que aquella que contiene $20 \%$ de grasa de res, debido principalmente al contenido de humedad que le imparte la pulpa de mango ( $82 \%$ de humedad). El contenido de grasa de la hamburguesa optimizada fue de 10.23 $\%$, una reducción del $48 \%$ respecto a la hamburguesa típica. La mayor parte de la grasa en la hamburguesa optimizada proviene de la adición de harina de linaza (31 \% de lípidos), lo que significa un mayor contenido de ácidos grasos poliinsaturados, principalmente ácido a-linolénico.

De acuerdo al perfil de ácidos grasos, mostrados en la Tabla 5, el contenido de ácidos grasos saturados y mono insaturados fue menor en la formulación óptima debido a un menor contenido de ácido palmítico (C16:0) y oleico (18:1 n9 cis), respectivamente. Además, se observa un mayor contenido de ácidos grasos poliinsaturados en la formulación óptima, principalmente por un aumento en el contenido de ácido linoleico (C18:2 n9 cis) y ácido linolénico (C18:3 n3), siendo este último el ácido graso principal de la familia omega-3. Se ha comprobado que cuando éste es consumido como parte de la dieta trae múltiples beneficios a la salud, destacando una disminución de las ECV (Yue et al., 2020). La hamburguesa optimizada puede ser más atractiva al consumidor al presentar un mejor perfil de ácidos grasos en su composición sin demérito en sus propiedades fisicoquímicas y sensoriales. 
Tabla 5. Análisis proximal y perfil de ácidos grasos de la hamburguesa optimizada y su comparación con una hamburguesa típica con $20 \%$ de grasa. Table 5. Proximal analysis and fatty acid profile of the optimized beef patties and comparison with a typical beef patty with $20 \%$ fat.

\begin{tabular}{|c|c|c|c|}
\hline & \multicolumn{3}{|c|}{ Tratamientos } \\
\hline & Testigo ( $20 \%$ grasa) & a Optimizada & Valor $p$ \\
\hline \multicolumn{4}{|c|}{ Análisis proximal } \\
\hline Humedad & $63.42 \pm 0.28$ & $67.91 \pm 0.28$ & $<0.001$ \\
\hline Grasa & $19.55 \pm 0.16$ & $10.23 \pm 0.12$ & $<0.001$ \\
\hline Proteína & $15.96 \pm 0.51$ & $17.04 \pm 0.64$ & 0.257 \\
\hline Ceniza & $1.48 \pm 0.04$ & $1.55 \pm 0.02$ & 0.267 \\
\hline \multicolumn{4}{|c|}{ Perfil de ácidos grasos } \\
\hline C12:0 & $0.08 \pm 0.01$ & $0.06 \pm 0.01$ & 0.395 \\
\hline C14:0 & $2.36 \pm 0.23$ & $1.46 \pm 0.03$ & 0.060 \\
\hline C15.0 & $0.25 \pm 0.02$ & $0.25 \pm 0.01$ & 0.977 \\
\hline C16:0 & $23.80 \pm 1.85$ & $15.36 \pm 0.00$ & 0.044 \\
\hline C17:0 & $0.68 \pm 0.07$ & $0.79 \pm 0.00$ & 0.245 \\
\hline C18:0 & $12.36 \pm 0.80$ & $11.14 \pm 0.15$ & 0.273 \\
\hline$\Sigma$ AGS & $39.52 \pm 2.95$ & $29.05 \pm 0.09$ & 0.041 \\
\hline C14:1 & $0.96 \pm 0.09$ & $0.26 \pm 0.01$ & 0.017 \\
\hline C15:1 & $0.42 \pm 0.03$ & $0.51 \pm 0.00$ & 0.098 \\
\hline C16:1 & $4.59 \pm 0.48$ & $1.64 \pm 0.03$ & 0.026 \\
\hline C17:1 & $0.66 \pm 0.06$ & $0.56 \pm 0.01$ & 0.234 \\
\hline C18:1 n9 cis & $42.82 \pm 2.48$ & $31.80 \pm 0.12$ & 0.047 \\
\hline$\Sigma$ AGM & $49.45 \pm 3.14$ & $34.78 \pm 0.16$ & 0.043 \\
\hline $\mathrm{C} 18: 2 \mathrm{n} 6$ cis & $4.14 \pm 1.27$ & $10.50 \pm 0.04$ & 0.037 \\
\hline C18:3 n3 & $0.38 \pm 0.00$ & $23.42 \pm 0.09$ & $<0.001$ \\
\hline C20:3 n6 & $0.52 \pm 0.01$ & $0.67 \pm 0.03$ & 0.029 \\
\hline$\Sigma$ AGP & $5.04 \pm 1.27$ & $34.60 \pm 0.16$ & 0.002 \\
\hline
\end{tabular}

AGS = ácidos grasos saturados, $\mathrm{AGM}=$ ácidos grasos monoinsaturados,

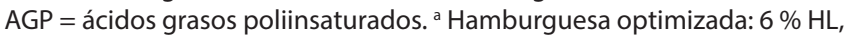
$10.5 \%$ PM y $3.5 \%$ CD.
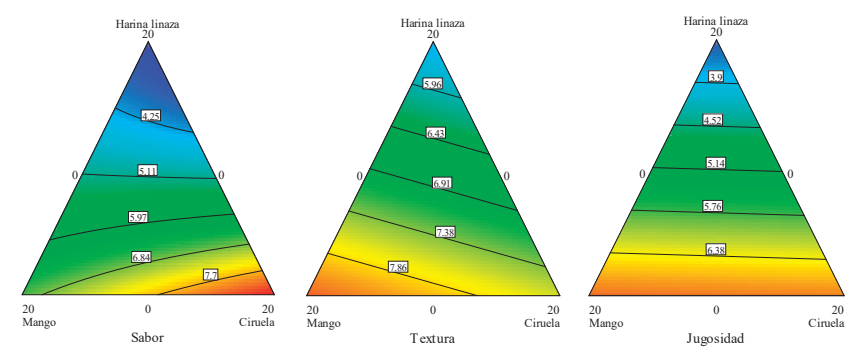

Figura 3. Gráfico de contorno de diseño de mezclas para atributos sensoriales: sabor, textura y jugosidad de hamburguesas de carne de bovino adicionadas con $\mathrm{HL}$, PM y CD.

Figure 3. Mixture design contour plot for sensory attributes: flavor, texture and juiciness of beef patty added with FS, MP and DP.

\section{CONCLUSIONES}

El detrimento en las propiedades fisicoquímicas y sensoriales observadas en estudios previos por la adición de harina de linaza en hamburguesas de carne de bovino fue disminuido por la inclusión de pulpa de mango y ciruela deshidratada. La combinación óptima de los ingredientes que resultó en un producto con propiedades fisicoquímicas y sensoriales aceptables fue de $6 \% \mathrm{HL}, 10.5 \%$ PM y $3.5 \%$ CD. El uso de pulpa de mango y ciruela deshidratada en hamburguesas de carne de bovino, permitió duplicar el contenido de harina de linaza reportado en investigaciones anteriores, además de aumentar el contenido de ácidos grasos poliinsaturados, principalmente ácido linoleico y ácido linolénico. La harina de linaza, pulpa de mango y ciruela deshidratada son ingredientes con gran potencial para el desarrollo de productos cárnicos con una connotación más saludable. Investigaciones para determinar los efectos en la salud del consumidor son necesarios. Es importante también precisar que este tipo de productos no son la solución a los problemas de salud derivados de una mala alimentación.

\section{AGRADECIMIENTOS}

Los autores agradecen al Consejo Nacional de Ciencia y Tecnología por la beca otorgada al primer autor durante sus estudios de Maestría en Ciencias.

\section{REFERENCIAS}

AOAC. (1990). Official Methods of Analysis. In Association of Analytical Chemists. 15th ed.). Washington, DC.

Baugreet, S., Kerry, J. P., Allen, P. , Hamill, R. M. 2017. Optimisation of protein-fortified beef patties targeted to the needs of older adults: a mixture design approach. Meat Science. 134: 111-118.

Bhat, A., Kumar, A., Sheikh, S., Dar, M. , Haq, Z. 2017. Utilisation of mango peel powder as phytopreservative in the refrigeration storage of chicken cutlets. International Journal of Livestock Research. 7: 90-99.

Bilek, A. E. , Turhan, S. 2009. Enhancement of the nutritional status of beef patties by adding flaxseed flour. Meat Science. 82: 472-477.

Botez, E., Nistor, O. V., Andronoiu, D. G., Mocanu, G. D. , Ghinea, I. O. (2017). Meat product reformulation: Nutritional benefits and effects on human health. In Functional Food-Improve Health through Adequate Food.). London (UK). Intech Open. 167-184.

Bourne, M. C. 1978. Texture profile analysis. Food Technolology. 32: 62-66.

Braña-Varela, D., Rodríguez, E., Lozano, M., Sanchez-Escalante, A., Torrescano-Urrutia, G., Arenas de Moreno, M., Partida de la Peña, J., Ponce-Alquicira, E. , Rios-Rincón, F. (2011). Manual de analisis de calidad en muestras de carne http://www. anetif.org/files/pages/0000000034/03-manual-de-analisisdecalidad-en-muestras-de-carne.pdf

Claus, J. R. (1995). Methods for the objective measurement of meat product texture. Paper presented at the Reciprocal Meat Conference Proceedings.

Cofrades, S., Serrano, A., Ayo, J., Solas, M. T., Carballo, J. , Colmenero, F. J. 2004. Restructured beef with different 
proportions of walnut as affected by meat particle size. European Food Research and Technology. 218: 230-236.

Comecarne. (2021). Consejo Mexicano de la Carne. Compendio estadístico 2021. En: https://comecarne.org/

do Prado, M., Queiroz, V., da Veiga Correia, V., Neves, E., Roncheti, E., Gonçalves, A., de Menezes, C. , de Oliveira, F. 2019. Physicochemical and sensorial characteristics of beef burgers with added tannin and tannin-free whole sorghum flours as isolated soy protein replacer. Meat Science. 150: 93-100.

Domínguez-Vara, I. , Mondragón-Ancelmo, J. 2018. Factores determinantes para el consumo de la carne de bovino en los estados de México y Michoacán. Avances de la Investigación Sobre Producción Animal y Seguridad Alimentaria en México: 1267.

Gill, S. K., Lever, E., Emery, P. W. , Whelan, K. 2019. Nutrient, fibre, sorbitol and chlorogenic acid content of prunes (Prunus domestica): an updated analysis and comparison of different countries of origin and database values. International Journal of Food Sciences and Nutrition. 70: 924-931.

Jarvis, N., O'Bryan, C. A., Ricke, S. C. , Crandall, P. G. 2015. The functionality of plum ingredients in meat products: A review. Meat Science. 102: 41-48.

Keenan, D. F., Resconi, V. C., Kerry, J. P. , Hamill, R. M. 2014. Modelling the influence of inulin as a fat substitute in comminuted meat products on their physico-chemical characteristics and eating quality using a mixture design approach. Meat Science. 96: 1384-1394.

Lebaka, V., Wee, Y., Ye, W. , Korivi, M. 2021. Nutritional composition and bioactive compounds in three different parts of mango fruit. International Journal of Environmental Research and Public Health. 18: 741.

Maldonado-Celis, M. E., Yahia, E. M., Bedoya, R., Landázuri, P., Loango, N., Aguillón, J., Restrepo, B. , Guerrero Ospina, J. C. 2019. Chemical composition of mango (Mangifera indica L.) fruit: Nutritional and phytochemical compounds. Frontiers in Plant Science. 10: 1073.

Nuñez de Gonzalez, M., Hafley, B., Boleman, R., Miller, R., Rhee, K. , Keeton, J. 2008. Antioxidant properties of plum concentrates and powder in precooked roast beef to reduce lipid oxidation. Meat Science. 80: 997-1004.

Nuñez de Gonzalez, M., Hafley, B., Boleman, R., Miller, R., Rhee, K. , Keeton, J. 2009. Qualitative effects of fresh and dried plum ingredients on vacuum-packaged, sliced hams. Meat Science. 83: 74-81.

Qiu, Z. Z., Chin, K. B. 2021. Physicochemical properties and shelflife of raw and cooked patties added with various levels of grape tomato powder by different drying methods. LWTFood Science and Technology. 146: 111415.

Reyes-Padilla, E., Valenzuela-Melendres, M., Camou, J. P., Sebranek, J. G., Alemán-Mateo, H., Dávila-Ramírez, J. L.,
Cumplido-Barbeitia, G. , González-Ríos, H. 2018. Quality evaluation of low fat bologna-type meat product with a nutritional profile designed for the elderly. Meat Science. 135: 115-122.

Selani, M. M., Shirado, G. A., Margiotta, G. B., Rasera, M. L., Marabesi, A. C., Piedade, S. M., Contreras-Castillo, C. J. , Canniatti-Brazaca, S. G. 2016. Pineapple by-product and canola oil as partial fat replacers in low-fat beef burger: Effects on oxidative stability, cholesterol content and fatty acid profile. Meat Science. 115: 9-15.

Serna-Cock, L., García-Gonzales, E. , Torres-León, C. 2016. Agro-industrial potential of the mango peel based on its nutritional and functional properties. Food Reviews International. 32: 364-376.

SIAP. (2021). Servicio de Información Agroalimentaria y Pesquera. Escenario mensual de productos agroalimentarios En: https://www.gob.mx/siap/documentos/escenarios-deproductos-agroalimentarios-266425.

Szerman, N., Ferrari, R., Sancho, A. M. , Vaudagna, S. 2019. Response surface methodology study on the effects of sodium chloride and sodium tripolyphosphate concentrations, pressure level and holding time on beef patties properties. LWT-Food Science and Technology. 109: 93-100.

Toldrá, F. 2003. Muscle foods: water, structure and functionality. Food Science and Technology International. 9: 173-177.

Torres-León, C., Rojas, R., Contreras-Esquivel, J. C., Serna-Cock, L., Belmares-Cerda, R. E. , Aguilar, C. N. 2016. Mango seed: Functional and nutritional properties. Trends in Food Science \& Technology. 55: 109-117.

Valenzuela-Melendres, M., Camou, J., Torrentera-Olivera, N., Álvarez-Almora, E., González-Mendoza, D., Avendaño-Reyes, L. , González-Ríos, H. 2014. Response surface methodology for predicting quality characteristics of beef patties added with flaxseed and tomato paste. Meat Science. 97: 54-61.

Valenzuela-Melendres, M., Camou, J., Torrentera-Olivera, N., Viuda-Martos, M. , González-Rios, H. 2018. Nutritional quality of beef patties with added flaxseed and tomato paste. CyTAJournal of Food. 16: 263-270.

Wongkaew, M., Sommano, S. R., Tangpao, T., Rachtanapun, P. , Jantanasakulwong, K. 2020. Mango peel pectin by microwave-assisted extraction and its use as fat replacement in dried Chinese sausage. Foods. 9: 450.

Yıldız-Turp, G. , Serdaroglu, M. 2010. Effects of using plum puree on some properties of low fat beef patties. Meat Science. 86: 896-900.

Yue, H., Qiu, B., Jia, M., Liu, W., Guo, X., Li, N., Xu, Z., Du, F., Xu, T. , Li, D. 2020. Effects of a-linolenic acid intake on blood lipid profiles: a systematic review and meta-analysis of randomized controlled trials. Critical Reviews in Food Science and Nutrition: 1-17. 